Revue internationale du CRIRES : innover dans la tradition de Vygotsky (2021) ISSN 2291-6717, vol 5, no 2, 95-111

Le développement professionnel autonome chez les enseignants dans le conteste de la pandémie

\title{
LE DÉVELOPPEMENT PROFESSIONNEL AUTONOME CHEZ LES ENSEIGNANTS DANS LE CONTEXTE DE LA PANDÉMIE
}

Viorica Dobrica-Tudor, PhD

Chercheuse indépendante

Alexandra Coutlée

Conseillère pédagogique

\author{
viorica.dobrica@umontreal.ca; tdviorica@yahoo.com
}

alexandracoutlee@gmail.com

\section{Résumé}

Lors de cette recherche nous avons exploré si les médiations d'ordre cognitif, social et technique, développées entre certains acteurs de l'éducation dans le contexte particulier d'un bouleversement des pratiques dû à la pandémie de COVID-19 ont débouché sur le développement professionnel chez les enseignants travaillant dans des écoles primaires et secondaires québécoises. Forcés d'enseigner à distance ou en mode hybride par une longue période de confinement, les enseignants ont dû garder le contact avec les élèves et assurer la continuité des apprentissages de ceux-ci. Ce contexte a amené les enseignants à se tourner vers différentes ressources de médiation, notamment vers les pairs par l'intermédiaire des réseaux sociaux et les conseillers pédagogiques. Opérationnalisé par des changements de pratiques dans le contexte d'une crise sanitaire sans précédent, le développement professionnel des 29 enseignants participants a été étudié par l'intermédiaire de la méthodologie Q. Les classements des énoncés élaborés à cette fin font ressortir trois profils d'enseignants : ceux dont le développement professionnel a reposé en majorité sur les interactions avec des collègues connus, les enseignants qui se sont plutôt fiés à leurs réflexions et aux conseillers pédagogiques et ceux qui se sont inspirés des échanges professionnels sur les réseaux sociaux pour développer leur pratique. Rapportés à l'enseignement dans des contextes particuliers, nos résultats pourraient servir aux conseillers pédagogiques pour mieux soutenir le développement de compétences professionnelles permettant l'adaptation des pratiques enseignantes à des situations hors du commun.

Mots-clés: développement professionnel, pandémie, profil des enseignants, réseaux sociaux, conseiller pédagogique

\begin{abstract}
Through this research we explored whether the cognitive, social and technical mediations developed between certain education actors, in the particular context of a disruption of practices due to the COVID-19 pandemic, have led to professional development among teachers working in elementary and secondary schools in Quebec. Forced to teach remotely or in hybrid mode by a long period of confinement, teachers had to keep in touch with students and ensure the continuity of their learning. This context has led teachers to turn to various mediation resources, such as through peers, social media or educational advisers. Operationalized by changes in practice in the
\end{abstract}


Revue internationale du CRIRES : innover dans la tradition de Vygotsky (2021) ISSN 2291-6717, vol 5, no 2, 95-111

Le développement professionnel autonome chez les enseignants dans le conteste de la pandémie

context of an unprecedented health crisis, the professional development of the 29 participating teachers was studied using the Q methodology. The classifications of the statements developed for this purpose highlight three teacher profiles: those whose professional development was mainly based on interactions with known colleagues, teachers who relied more on their reflections and pedagogical advisers and those who were inspired by professional exchanges on social media to develop their practice. Related to teaching in specific contexts, our results could be used by pedagogical advisers to better support the development of professional skills allowing the adaptation of teaching practices to unusual situations.

Keywords: professional development, pandemic, teacher profile, social media, pedagogical advisor

Toute correspondance concernant cet article doit être adressée à Viorica Dobrica-Tudor (tdviorica@yahoo.com) 
Revue internationale du CRIRES : innover dans la tradition de Vygotsky (2021) ISSN 2291-6717, vol 5, no 2, 95-111 Le développement professionnel autonome chez les enseignants dans le conteste de la pandémie

\section{Problématique et cadre de référence}

Au sens sociologique, une crise représente une période de difficulté, une «[...] situation anomique de perturbations affectant finalement le système social dans son ensemble » (Ferry, 2014, p.52). La pandémie due à la COVID-19, par les bouleversements qu'elle a apportés à l'ensemble du système social, a provoqué des transformations inédites en générant une crise majeure. Bien entendu, le milieu éducatif n'y a pas échappé et la façon de dispenser l'enseignement chez les élèves du primaire et du secondaire nous apparaît comme le facteur ayant subi les changements les plus importants. Ainsi, plus de 1,5 milliard d'apprenants de 165 pays ont vécu des expériences inédites d'apprentissage en raison «des précautions et des politiques mises en œuvre pour endiguer la propagation de la maladie » (Duval et al., 2021, p. 2). Pour leur part, les écoles québécoises, elles ont dû cesser les activités en présence en mars 2020. Par la suite, certaines écoles ont repris les cours en ligne, d'autres en alternance et finalement en présence avec plusieurs mesures sanitaires à appliquer.

Selon plusieurs auteurs, parmi lesquels nous pouvons mentionner Stone et Springer (2019), l'apprentissage à distance nécessite une approche et des compétences différentes de l'enseignement en présence, des ressources et du soutien de la part des directions et des collègues ; des nouvelles façons d'agir compétent qui renvoient au développement professionnel. Étant donné tous ces changements, il nous semble pertinent de se demander quelles sont leurs conséquences sur les pratiques professionnelles, particulièrement sur le développement professionnel des enseignants. Par conséquent, notre question de recherche consiste à savoir si les médiations d'ordre cognitif, social et technique, relevées entre des acteurs de l'éducation dans le contexte de confinement imposé par la pandémie de COVID-19, ont abouti vers le développement professionnel chez les enseignants travaillant dans des écoles primaires et secondaires québécoises.

Mis devant une situation inédite qui leur imposait une rupture brusque avec la forme scolaire classique, l'enseignement présentiel, les enseignants se sont tournés vers plusieurs ressources. Parmi celles-ci, nous nous penchons principalement sur le rôle des réseaux sociaux et des conseillers pédagogiques. Selon Macià et García (2016), c'est notamment le potentiel de créer une proximité spatiale et temporelle qui explique pourquoi la participation de nombreux enseignants à des communautés en ligne est devenue une activité courante. En s'engageant dans des interactions collaboratives, via les plateformes de médias sociaux, les enseignants apprennent, communiquent avec leurs pairs et partagent des matériels pédagogiques et didactiques, malgré la distance géographique (Carpenter \& Krutka, 2015). Dans ce contexte, il n'est pas surprenant que les réseaux sociaux comme Facebook, Twitter, WhatsApp, YouTube et Instagram soient devenus le refuge des enseignants, étudiants et élèves tout au long de la période de la pandémie (Ghouname, 2020). À titre d'exemple, Shriki (2021) conclut que la fermeture des écoles a expliqué à la fois la croissance rapide du nombre d'enseignants en mathématiques qui utilisaient la plateforme Facebook et les raisons pour lesquelles les enseignants utilisaient la plateforme. Ces derniers sont passés de l'échange de matériel didactique aux réflexions de groupe sur des stratégies d'enseignement à distance. D'autres enseignants, en cherchant des astuces pour mieux vivre la situation, se sont réunis dans de nouveaux sous-groupes. C'est le cas du sous-groupe professionnel «Le Monde de l'éducation », créé mai 2020, dont le nombre de participants aux échanges est arrivé à 11292 au mois d'août 2021. Ce sont des exemples d'actions qui témoignent de la volonté de partager des connaissances et des méthodes d'enseignement sur les réseaux sociaux et que nous, en concordance avec Liljekvist et al., (2021), associons au développement professionnel chez les enseignants. 
Revue internationale du CRIRES : innover dans la tradition de Vygotsky (2021) ISSN 2291-6717, vol 5, no 2, 95-111 Le développement professionnel autonome chez les enseignants dans le conteste de la pandémie

Pour ce qui est des conseillers pédagogiques, ils agissent en tant qu'agents de développement professionnel des enseignants dans une perspective de formation continue. Pour ce faire, ils interviennent « directement auprès des enseignants, novices ou experts, » en proposant des solutions aux pratiques problématiques, des outils et des ressources pédagogiques, etc. (Duchesne, 2016, p. 635). À leur tour, en reconnaissant l'apport de leur travail à la transformation des pratiques pédagogiques, Tardif et Lessard (2004) les appellent des agents de changement des pratiques. Pour sa part, Rioux-Dolan (2004) conçoit l'accompagnement des enseignants par un conseiller pédagogique comme un appui mutuel dans le but de trouver ensemble les meilleures solutions « sans exclure qu'une personne puisse agir comme guide et comme soutien pour l'autre » (p.20). Pour remplir leurs rôles charnières durant la crise sanitaire, les conseillers pédagogiques ont dû faire " preuve de créativité et ont su innover pour développer des moyens de réseautage, des plates-formes d'accès aux savoirs, des modalités d'enseignement à distance, etc. » (Granger et Guillemette, 2021, p. 33). Sollicités durant cette période sur un modèle d'enseignement qu'ils connaissaient eux-mêmes assez peu, ils se sont retrouvés dans la posture d'être sollicités à la dernière minute, donc de répondre dans un laps de temps relativement court. En plus des rencontres face à face, ils ont dû faire appel aux solutions numériques telles que les courriels et les visioconférences pour mieux répondre aux besoins des enseignants. Selon une étude menée par Granger et Guillemette (2021) auprès de 85 conseillers pédagogiques, seuls $27 \%$ des répondants ont été en mesure d'accompagner les enseignants en présentiel, alors que $31 \%$ l'ont fait en ligne et $42 \%$ ont alterné entre un modèle en présentiel et à distance.

Les compétences développées ou renforcées à la suite des interactions professionnelles avec des pairs et des conseillers pédagogiques dans le but d'adapter les pratiques et de sortir de la routine sont associées par Liou et Canrinus (2020) au développement professionnel. Selon les chercheurs, le processus de développement professionnel va de pair avec l'apprentissage professionnel lorsqu'il s'agit de la pratique des enseignants; un processus qui demande du temps et entraine de l'engagement dans des relations humaines afin de renforcer les compétences.

Dans le même sens, Lefeuvre, Garcia et Namolovan (2009) affirment que le développement professionnel réside dans un processus d'acquisition de savoirs professionnels qui influencent l'évolution des pratiques et les modes de pensée des acteurs. La définition du développement professionnel sur laquelle repose cette étude ressort des travaux de (Lameul et al.,) qui le décrivent comme un «processus individuel, structuré socialement, de changement de pratiques et d'acquisition progressive de compétences reconnues par la personne elle-même et par la communauté professionnelle dans laquelle la personne prend une part active et s'engage » (p. 102). En concordance avec cette définition, nous envisageons le processus de développement professionnel conditionné par l'engagement des enseignants dans des réflexions et des interactions professionnelles afin d'acquérir de nouvelles compétences qui leur permettront d'opérer des changements de pratiques.

En s'engageant dans des relations de travail avec les pairs et les conseillers pédagogiques, les enseignants s'engagent dans une forme de médiation qui désigne le savoir comme une production humaine socialement déterminée qui requiert un processus cognitif d'objectivation (Lenoir, 1996). Pour sa part, Berten (1999, p. 38) parlait d'un « réagencement des deux modes de médiations, symbolique et technique ». Pour lui, le mode symbolique, c'est-à-dire le langage, fait partie de l'essence humaine et celui technique est matériel, instrumental et placé à l'extérieur. Quant à eux, Durat et Kern (2019) ont interprété la médiation comme un concept pluridimensionnel qui compte trois dimensions: une dimension cognitive, qui pointe le raisonnement fait en situation, une autre conative, qui vise l'activité et les limites des actions 
menées, et une troisième socioaffective, qui fait ressortir la dynamique relationnelle et le retentissement subjectif pour la personne. Mashhadi et al., (2020) ajoutent qu'il y a des liens entre la médiation dans une communauté de partage des connaissances et le développement professionnel des enseignants. De notre côté, lors de cette étude, nous nous joignons plus à la théorie de Desgagné et Bednarz (2005, p. 254) pour lesquels « la médiation prend [...] forme à travers la mise à contribution des différents points de vue des acteurs engagés dans un diagnostic collectif, ouvrant sur des pistes d'action possibles, voire l'exploration de solutions concrètes et en contexte ».

En résumé, si les échanges professionnels en présentiel ont perdu de l'importance ou même ont été interrompus à cause de la crise sanitaire, les réflexions et les partages d'idées et de matériel avec des collègues et les conseillers pédagogiques, via des plateformes sociales, sont devenus des sources importantes d'inspiration et de médiation; des médiations centrées sur le développement des compétences spécifiques à une situation particulière, voire la crise pandémique.

\section{Méthode}

La méthodologie Q, choisie pour traiter les données de cette recherche, a été conçue par Stephenson (1935) dans le but de révéler des modèles de subjectivité, tels que les croyances, les sentiments, les points de vue et les attitudes (Ellingsen et al., 2012). La technique consiste à proposer le classement, d'après une échelle imposée, d'un nombre d'énoncés dont l'analyse dévoile une représentation statistique des attitudes individuelles (Vérin \& Peterfalvi, 1985). À la différence de l'approche $\mathrm{R}$, où les participants cotent sur une échelle Likert les énoncés séparément les uns des autres, dans l'approche Q, la distribution forcée, imposée par la structure de la fiche de réponses, les amène à envisager et à prioriser les énoncés les uns par rapport aux autres (Gauzente, 2013).

Sur le plan opérationnel, nous avons structuré la collecte et l'étude des données en trois étapes : l'élaboration des énoncés (l'échantillon Q) et de la fiche de réponses; la sélection des participants (l'échantillon P); l'analyse et l'interprétation des données.

\section{L'échantillon $Q$ et la collecte des données}

Pour concevoir les énoncés, nous nous sommes inspirés des écrits qui portent sur l'utilisation des réseaux sociaux, dont la plupart se retrouvent dans la liste de références, et d'entretiens avec quatre enseignantes. Les 34 énoncés élaborés, dont 31 se retrouvent en tant qu'énoncés distinctifs des trois facteurs, soulèvent des réflexions sur le développement professionnel des enseignants des écoles primaires et secondaires avant, durant et après la pandémie due à la COVID-19. Après l'élaboration des énoncés, nous avons envoyé à chaque participant par voie électronique le formulaire de consentement et un bref questionnaire. Le formulaire de consentement indiquait les conditions de participation, le respect de l'anonymat et le droit de se retirer à tout moment sans aucune conséquence. Le questionnaire sollicitait des réponses relatives à l'ordre d'enseignement, à l'expérience totale en enseignement et à celle dans la tâche actuelle. Dans un deuxième temps, ils ont reçu la fiche de consignes, la feuille avec les énoncés et la fiche de réponses.

Après la signature du formulaire de consentement, les participants ont rempli la fiche de réponses (ils ont opéré les Q-sorts) en inscrivant dans chaque case, un seul chiffre, de 1 à 34. Chaque nombre correspondait à un énoncé distinct de la feuille d'énoncés, et il devait être utilisé une seule fois. Pour ce faire, ils ont dû prendre en considération les consignes présentées dans la 
fiche de consignes et la structure de la fiche de réponses (voir Figure 1) et réfléchir jusqu'à quel point (de -4 , le moins important, jusqu'à la valeur +4 , le plus important) chaque énoncé reflétait chez eux une façon de faire avant, durant et après la période de pandémie :

\section{Figure 1}

La fiche de réponses

\begin{tabular}{|c|c|c|c|c|c|c|c|c|}
\hline $\begin{array}{l}\stackrel{-4}{\text { (Le moins }} \\
\text { important) }\end{array}$ & -3 & -2 & -1 & $\begin{array}{c}0 \\
\text { (Plus ou } \\
\text { moins } \\
\text { important) }\end{array}$ & 1 & 2 & 3 & $\begin{array}{c}4 \\
\text { (Le plus } \\
\text { important) }\end{array}$ \\
\hline & & & & & & & & \\
\hline & & & & & & & & \\
\hline & & & & & & & & \\
\hline & & & & & & & & \\
\hline & & & & & & & & \\
\hline
\end{tabular}

\section{L'échantillon P}

Notre échantillon était un échantillon non probabiliste, composé de 29 enseignants œuvrant dans des classes régulières ou en adaptation scolaire des écoles primaires (neuf participants) et secondaires (vingt participants) québécoises. Tous les participants avaient au moment de la participation à l'étude, soit au printemps 2021, au moins deux ans d'expérience professionnelle, ce qui indique qu'ils ont commencé leur enseignement avant la pandémie, donc qu'ils ont enseigné autant en mode présentiel que virtuel ou hybride. Notre échantillon compte des enseignants, dont les années d'ancienneté en enseignement, autant totale que dans la tâche actuelle, couvre de manière satisfaisante la probabilité d'offrir un large éventail de points de vue sur le développement professionnel durant la pandémie.

\section{L'analyse factorielle Q}

Les classements des participants (les Q-sorting) ont été traités au moyen d'une analyse factorielle à l'aide du logiciel PQMethod, un logiciel libre créé à partir des formules proposées par Brown (1980). L'analyse des classements des énoncés sera explorée en deux temps : la sélection des facteurs et la constitution de la matrice de corrélation factorielle (la matrice des contributions des Q-sorts aux facteurs), suivie par l'analyse par facteurs.

\section{La sélection des facteurs et la matrice de corrélation factorielle}

Chaque facteur met en lumière un point de vue unique sur le thème de la recherche (Ramlo \& John, 2020); il est représenté par un regroupement « composé de participants qui ont classé les énoncés d'une manière identique » (Dariel, 2013, p. 58). Les écrits recommandent en principal deux critères pour la sélection du nombre des facteurs, soit la valeur propre supérieure à 1,00 et un minimum de deux sorts représentatifs pour chaque facteur. Une fois prise la décision relative au 
nombre de facteurs, le logiciel élabore la matrice des contributions factorielles, autrement dit, la matrice des $Q$-sorts aux facteurs.

La matrice initiale indique « les saturations (corrélations) de chaque tri pour chacun des facteurs retenus pour l'analyse [...], la valeur propre du facteur et la part de la variance totale expliquée par les facteurs » (Kuhne et al., 2008, p. 49). Les tris représentatifs du facteur, c'est-àdire les tris qui le représentent, expliquent au moins la moitié de sa variance et sont marqués d'un «X » par le logiciel. La variance expliquée par les trois facteurs représente presque $60 \%$ de la variance totale, ce qui est acceptable pour une recherche qui explore un large éventail de réflexions sur un métier complexe, comme l'enseignement. Les valeurs des coefficients de fiabilité composite, soit 0,970 et 0,976, indiquent des niveaux élevés de cohérence interne de chaque facteur (voir l'Annexe).

Notre analyse factorielle repose sur les valeurs des tris de synthèse des énoncés distinctifs des trois facteurs retenus pour analyse. Un tri de synthèse représente le résultat « du tri qu'aurait opéré un sujet idéal, parfaitement représentatif du facteur » (Kuhne et al., 2008, p. 51). Les tris représentatifs de chaque facteur sont significatifs au seuil de $\mathrm{p}<.05$ ou de $\mathrm{p}<.01$; ces derniers sont marqués avec un astérisque (*).

\section{L'analyse des énoncés représentatifs du Facteur 1}

Selon les énoncés représentatifs de ce facteur (voir Tableau 1), les huit participants qui les ont opérés se caractérisent par des niveaux élevés de confiance dans les solutions que l'Internet offre à leurs problèmes d'ordre technique (énoncé 19) et par des échanges avec des collègues proches sur la gestion de classe et des aspects de nature pédagogique (énoncés 21 et 22). Par ailleurs, il faut mentionner les valeurs négatives des tris de synthèse des énoncés qui visent la recherche des solutions aux problèmes de gestion de classe sur les réseaux sociaux (énoncés 6 et 13) dans les trois facteurs. Cela signifie que pour résoudre ce genre de problèmes, les enseignants préfèrent les réflexions avec des collègues proches. Au contraire, les énoncés 19 et 22 sont positifs dans tous les facteurs, ce qui suggère que les participants font confiance aux solutions offertes par l'Internet à leurs problèmes techniques et par les collègues aux questions d'ordre pédagogique.

Sans que l'engagement dans des parcours d'autoformation (énoncé 33) ou la participation sur les réseaux sociaux durant la pandémie fassent partie de leurs options de développement professionnel (énoncé 12), ils n'envisagent pas devenir plus actifs ou de se brancher plus après la période de pandémie (énoncés 16; 15). Avant la pandémie, les réseaux sociaux étaient utilisés pour des solutions d'ordre pédagogique (énoncé 5), sans toutefois être considérés comme une source d'inspiration essentielle (énoncé 2), leur influence sur les pratiques n'a pas augmenté durant la pandémie et baissera après la pandémie : durant la pandémie, ils ne se sont pas montrés plus actifs sur les réseaux sociaux et ne les ont pas utilisés plus souvent pour échanger sur des démarches pédagogiques ou pour venir en aide aux collègues (énoncés $8 ; 12 ; 14 ; 9$ ).

Pour ce qui est des relations avec les conseillers pédagogiques, les enseignants de ce profil apprécient les accompagnements individualisés, préférablement en présentiel, et les réponses rapides à leurs questions (énoncés 28; 29; 30; 32).

En résumé, le premier facteur regroupe des enseignants pour lesquels les solutions aux problèmes techniques trouvées sur l'Internet, les échanges avec des collègues proches et les accompagnements individualisés de la part des conseillers pédagogiques ont été plus importants durant la crise pandémique que les interactions professionnelles sur des réseaux sociaux. 
Revue internationale du CRIRES : innover dans la tradition de Vygotsky (2021) ISSN 2291-6717, vol 5, no 2, 95-111 Le développement professionnel autonome chez les enseignants dans le conteste de la pandémie

\section{Tableau 1}

Les énoncés distinctifs du Facteur 1 et leurs scores de synthèse

\begin{tabular}{|c|c|c|c|c|}
\hline \multirow[t]{2}{*}{ No. } & \multirow[t]{2}{*}{ Énoncés } & \multicolumn{3}{|c|}{$\begin{array}{l}\text { Tris de } \\
\text { synthèse }\end{array}$} \\
\hline & & I & II & III \\
\hline 19 & Pour toutes questions d'ordre technique, je cherche sur Internet & 4* & 1 & 0 \\
\hline 5 & $\begin{array}{l}\text { Avant la pandémie, j'utilisais les réseaux sociaux pour des questions } \\
\text { pédagogiques }\end{array}$ & 2 & -3 & 1 \\
\hline 8 & $\begin{array}{l}\text { Durant la pandémie, je n'ai pas fait appel aux réseaux sociaux plus souvent } \\
\text { qu'avant }\end{array}$ & $2 *$ & -1 & -4 \\
\hline 21 & $\begin{array}{l}\text { Pour toutes questions en lien avec la gestion de classe, je m'adresse à des } \\
\text { gens que je connais }\end{array}$ & 2 & 4 & 4 \\
\hline 28 & $\begin{array}{l}\text { Je préfère avoir des accompagnements individualisés avec mon conseiller } \\
\text { pédagogique }\end{array}$ & 1 & 0 & -1 \\
\hline 22 & Pour les questions du domaine de la pédagogie, je m'adresse à un collègue & $\mathbf{1}^{*}$ & 4 & 4 \\
\hline 15 & $\begin{array}{l}\text { Après la pandémie, j'ai l'intention d'utiliser moins souvent les réseaux } \\
\text { sociaux }\end{array}$ & $1^{*}$ & -2 & -4 \\
\hline 26 & $\begin{array}{l}\text { Les meilleures solutions à mes problèmes en enseignement proviennent de } \\
\text { mes propres réflexions }\end{array}$ & $\mathbf{0}^{*}$ & 2 & -2 \\
\hline 10 & $\begin{array}{l}\text { Durant la pandémie, j'ai utilisé les réseaux sociaux pour régler les } \\
\text { problèmes techniques que je rencontrais en utilisant des outils numériques }\end{array}$ & $\mathbf{0}^{*}$ & -2 & 2 \\
\hline 30 & $\begin{array}{l}\text { J'apprécie quand le conseiller pédagogique prend le temps de me } \\
\text { rencontrer en présentiel }\end{array}$ & $\mathbf{0}$ & 1 & -2 \\
\hline 32 & J'apprécie quand mon conseiller me donne des réponses immédiates & $\mathbf{0}$ & 1 & 0 \\
\hline 14 & $\begin{array}{l}\text { Durant la pandémie, j'ai utilisé les réseaux sociaux pour des questions } \\
\text { pédagogiques }\end{array}$ & -1 & 0 & 3 \\
\hline 9 & $\begin{array}{l}\text { Durant la pandémie j'ai utilisé les réseaux sociaux pour venir en aide à mes } \\
\text { collègues plus souvent qu'avant la pandémie }\end{array}$ & $-1^{*}$ & 3 & 1 \\
\hline 34 & $\begin{array}{l}\text { J'aimerais pouvoir poser des questions en tout temps à un conseiller } \\
\text { pédagogique }\end{array}$ & $-1^{*}$ & 0 & 0 \\
\hline 23 & $\begin{array}{l}\text { Pour les questions du domaine de la pédagogie, je m'adresse à un conseiller } \\
\text { pédagogique }\end{array}$ & -1 & 1 & -1 \\
\hline 2 & $\begin{array}{l}\text { Avant la pandémie, les réseaux sociaux représentaient une source } \\
\text { d'inspiration essentielle }\end{array}$ & $-2^{*}$ & -3 & 1 \\
\hline 1 & Avant la pandémie, j'utilisais un réseau social de façon sporadique & -2 & -1 & -3 \\
\hline 12 & Durant la pandémie, je suis devenue très actif/active sur les réseaux sociaux & $-2 *$ & 0 & -1 \\
\hline 33 & $\begin{array}{l}\text { J'apprécie suivre des parcours d'autoformation et poser des questions à } \\
\text { mon conseiller pédagogique au besoin }\end{array}$ & $-2^{*}$ & 0 & 1 \\
\hline 29 & $\begin{array}{l}\text { J'apprécie quand le conseiller pédagogique prend le temps de me } \\
\text { rencontrer en virtuel }\end{array}$ & $-3^{*}$ & 2 & 0 \\
\hline 11 & $\begin{array}{l}\text { C'est durant la pandémie que j'ai commencé à utiliser les réseaux sociaux } \\
\text { pour découvrir des outils numériques }\end{array}$ & $-4^{*}$ & -1 & -2 \\
\hline
\end{tabular}


Revue internationale du CRIRES : innover dans la tradition de Vygotsky (2021) ISSN 2291-6717, vol 5, no 2, 95-111

Le développement professionnel autonome chez les enseignants dans le conteste de la pandémie

\begin{tabular}{|c|l|c|c|c|}
\hline 13 & $\begin{array}{l}\text { Durant la pandémie, j'ai utilisé les réseaux sociaux pour des questions sur } \\
\text { la gestion de classe }\end{array}$ & $\mathbf{- 4 *}$ & -1 & -2 \\
\hline 16 & $\begin{array}{l}\text { Après la pandémie, je vais augmenter la fréquence à laquelle j'utilise les } \\
\text { réseaux sociaux }\end{array}$ & $\mathbf{- 4 *}$ & -2 & -1 \\
\hline
\end{tabular}

\section{L'analyse des énoncés représentatifs du Facteur 2}

Huit enseignants ont opéré des tris représentatifs du deuxième facteur. Selon les énoncés les plus représentatifs (voir Tableau 2), c'est le profil des enseignants qui misent notamment sur leurs réflexions (énoncé 26) et sur les rencontres, préférablement virtuelles, avec des conseillers pédagogiques pour trouver les meilleures solutions à leurs problèmes de pratique durant la crise sanitaire (énoncés $29 ; 30 ; 23 ; 28)$. Les valeurs de plusieurs énoncés distinctifs du facteur $(3 ; 5 ; 2$; 4) relèvent leur manque d'intérêt face aux réseaux sociaux avant la pandémie et cela même s'il s'agissait des interactions avec des personnes ciblées ou de groupes restreints (énoncés 7). Pour ce qui est de l'utilisation comparative des réseaux sociaux durant et après la pandémie, ils ont été utilisés plus souvent qu'avant cette période en raison des questions d'ordre pédagogiques et seront encore utilisés une fois la crise sanitaire terminée (énoncés $8 ; 14 ; 15$ ).

Quant à leurs relations avec un conseiller pédagogique, elles semblent appréciées ; pour chercher des solutions aux problèmes d'ordre pédagogiques, ils se montrent disposés à participer aux rencontres en présentiel ou bénéficier d'accompagnements individualisés (énoncés 23 et 28). Par ailleurs, les interactions avec les conseilleurs pédagogiques représentent leur forme privilégiée d'échanges professionnels, au détriment de ceux avec les collègues et ceux sur les réseaux sociaux, ignorés avant et pas assez explorés durant la période de crise sanitaire.

Pour définir le profil de ces enseignants, il faut mentionner leur confiance dans leurs réflexions personnelles et les interactions avec les conseillers pédagogiques qu'ils privilégient lors de la recherche de solutions à leurs problèmes d'enseignement.

\section{Tableau 2}

Les énoncés distinctifs du Facteur 2 et leurs scores de synthèse

\begin{tabular}{|c|c|c|c|c|}
\hline \multirow[t]{2}{*}{ No. } & \multirow[t]{2}{*}{ Énoncés } & \multicolumn{3}{|c|}{$\begin{array}{l}\text { Tris de } \\
\text { synthèse }\end{array}$} \\
\hline & & $\mathrm{I}$ & II & III \\
\hline 26 & $\begin{array}{l}\text { Les meilleures solutions à mes problèmes en enseignement proviennent } \\
\text { de mes propres réflexions }\end{array}$ & 0 & $2^{*}$ & -2 \\
\hline 29 & $\begin{array}{l}\text { J'apprécie quand le conseiller pédagogique prend le temps de me } \\
\text { rencontrer en virtuel }\end{array}$ & 3 & $2^{*}$ & -2 \\
\hline 23 & $\begin{array}{l}\text { Pour les questions du domaine de la pédagogie, je m'adresse à un } \\
\text { conseiller pédagogique }\end{array}$ & -1 & $1^{*}$ & -1 \\
\hline 30 & $\begin{array}{l}\text { J'apprécie quand le conseiller pédagogique prend le temps de me } \\
\text { rencontrer en présentiel }\end{array}$ & 0 & 1 & -2 \\
\hline 14 & $\begin{array}{l}\text { Durant la pandémie, j'ai utilisé les réseaux sociaux pour des questions } \\
\text { pédagogiques }\end{array}$ & -1 & 0 & 3 \\
\hline
\end{tabular}


Revue internationale du CRIRES : innover dans la tradition de Vygotsky (2021) ISSN 2291-6717, vol 5, no 2, 95-111

Le développement professionnel autonome chez les enseignants dans le conteste de la pandémie

\begin{tabular}{|c|l|c|c|c|}
\hline 28 & $\begin{array}{l}\text { Je préfère avoir des accompagnements individualisés avec mon conseiller } \\
\text { pédagogique }\end{array}$ & 1 & $\mathbf{0}$ & -1 \\
\hline 1 & Avant la pandémie, j'utilisais un réseau social de façon sporadique & -2 & $\mathbf{- 1}$ & -3 \\
\hline 11 & $\begin{array}{l}\text { C'est durant la pandémie que j'ai commencé à utiliser les réseaux sociaux } \\
\text { pour découvrir des outils numériques }\end{array}$ & -4 & $\mathbf{- 1}^{*}$ & -2 \\
\hline 13 & $\begin{array}{l}\text { Durant la pandémie, j'ai utilisé les réseaux sociaux pour des questions sur } \\
\text { la gestion de classe }\end{array}$ & -3 & $\mathbf{- 1}^{*}$ & -3 \\
\hline 8 & $\begin{array}{l}\text { Durant la pandémie, je n'ai pas fait appel aux réseaux sociaux plus } \\
\text { souvent qu'avant }\end{array}$ & 2 & $\mathbf{- 1}^{*}$ & -4 \\
\hline 15 & $\begin{array}{l}\text { Après la pandémie, j'ai l'intention d'utiliser moins souvent les réseaux } \\
\text { sociaux }\end{array}$ & 1 & $\mathbf{- 2}^{*}$ & -4 \\
\hline 10 & $\begin{array}{l}\text { Durant la pandémie, j'ai utilisé les réseaux sociaux pour régler les } \\
\text { problèmes techniques que je rencontrais en utilisant des outils numériques }\end{array}$ & 0 & $\mathbf{- 2}^{*}$ & 2 \\
\hline 7 & $\begin{array}{l}\text { Avant la pandémie, je préférais les échanges sur les réseaux avec des } \\
\text { personnes ciblées ou des groupes restreints }\end{array}$ & 1 & $\mathbf{- 2}^{*}$ & 0 \\
\hline 4 & $\begin{array}{l}\text { Avant la pandémie, j'utilisais les réseaux sociaux pour découvrir de } \\
\text { nouveaux outils numériques }\end{array}$ & 4 & $\mathbf{- 3}^{*}$ & 3 \\
\hline 2 & $\begin{array}{l}\text { Avant la pandémie, les réseaux sociaux représentaient une source } \\
\text { d'inspiration essentielle }\end{array}$ & 2 & $\mathbf{- 3}^{*}$ & 1 \\
\hline 5 & $\begin{array}{l}\text { Avant la pandémie, j'utilisais les réseaux sociaux pour des questions } \\
\text { pédagogiques }\end{array}$ & 2 & $\mathbf{- 3}^{*}$ & 1 \\
\hline 3 & $\begin{array}{l}\text { Avant la pandémie, j'utilisais les réseaux sociaux pour régler les } \\
\text { problèmes techniques que je rencontrais en utilisant des outils numériques }\end{array}$ & 0 & $\mathbf{- 4}^{*}$ & 0 \\
\hline
\end{tabular}

\section{L'analyse des énoncés représentatifs du Facteur 3}

On peut caractériser brièvement les dix enseignants qui ont opéré des tris représentatifs du troisième facteur (voir Tableau 3 ) en les appelant « les utilisateurs actifs des réseaux sociaux », et cela à partir des énoncés le plus représentatif (énoncés 14 et 10).

Convaincus que les réseaux sociaux représentent l'avenir dans le monde de l'éducation et qu'ils sont une source d'inspiration significative (énoncés 18 et 2), ils leur attribuent plus d'importance qu'à d'autres sources d'information et même aux réflexions personnelles (énoncés 26; 20; 27).

\section{Tableau 3}

Les énoncés distinctifs du Facteur 3 et leurs scores de synthèse

\begin{tabular}{|c|l|c|c|c|}
\hline No. & \multicolumn{1}{|c|}{ Énoncés } & \multicolumn{3}{|c|}{$\begin{array}{c}\text { Tris de } \\
\text { synthèse }\end{array}$} \\
\cline { 3 - 5 } & & I & II & II \\
\hline 14 & $\begin{array}{l}\text { Durant la pandémie, j'ai utilisé les réseaux sociaux pour des questions } \\
\text { pédagogiques }\end{array}$ & -1 & 0 & $\mathbf{3}^{*}$ \\
\hline 10 & $\begin{array}{l}\text { Durant la pandémie, j'ai utilisé les réseaux sociaux pour régler les } \\
\text { problèmes techniques que je rencontrais en utilisant des outils numériques }\end{array}$ & 0 & -2 & $\mathbf{2}^{*}$ \\
\hline
\end{tabular}


Revue internationale du CRIRES : innover dans la tradition de Vygotsky (2021) ISSN 2291-6717, vol 5, no 2, 95-111

Le développement professionnel autonome chez les enseignants dans le conteste de la pandémie

\begin{tabular}{|c|l|c|c|c|}
\hline 17 & $\begin{array}{l}\text { Une fois la période de pandémie finie, je garderai la même fréquence } \\
\text { d'utilisation des réseaux sociaux }\end{array}$ & 0 & $\mathbf{2}$ \\
\hline 18 & $\begin{array}{l}\text { Je vois l'utilisation des réseaux sociaux comme l'avenir dans le monde de } \\
\text { l'éducation }\end{array}$ & -1 & -1 & $\mathbf{2}^{*}$ \\
\hline 2 & $\begin{array}{l}\text { Avant la pandémie, les réseaux sociaux représentaient une source } \\
\text { d'inspiration essentielle }\end{array}$ & -2 & -3 & $\mathbf{1}^{*}$ \\
\hline 5 & $\begin{array}{l}\text { Avant la pandémie, j'utilisais les réseaux sociaux pour des questions } \\
\text { pédagogiques }\end{array}$ & 2 & -3 & $\mathbf{1}$ \\
\hline 29 & $\begin{array}{l}\text { J'apprécie quand le conseiller pédagogique prend le temps de me } \\
\text { rencontrer en virtuel }\end{array}$ & -3 & 2 & $\mathbf{0}^{*}$ \\
\hline 23 & $\begin{array}{l}\text { Pour les questions du domaine de la pédagogie, je m'adresse à un } \\
\text { conseiller pédagogique }\end{array}$ & -1 & 1 & $\mathbf{- 1}$ \\
\hline 27 & $\begin{array}{l}\text { Pour choisir la meilleure stratégie, je fais une synthèse des informations } \\
\text { issues des plusieurs sources }\end{array}$ & 3 & 2 & $\mathbf{- 1}$ \\
\hline 28 & $\begin{array}{l}\text { Je préfère avoir des accompagnements individualisés avec mon conseiller } \\
\text { pédagogique }\end{array}$ & 1 & 0 & $\mathbf{- 1}$ \\
\hline 20 & Pour toutes questions d'ordre pédagogique, je cherche sur Internet & 3 & 3 & $\mathbf{- 2}$ \\
\hline 30 & $\begin{array}{l}\text { J'apprécie quand le conseiller pédagogique prend le temps de me } \\
\text { rencontrer en présentiel }\end{array}$ & 0 & 1 & $\mathbf{- 2}^{*}$ \\
\hline 26 & $\begin{array}{l}\text { Les meilleures solutions à mes problèmes en enseignement proviennent } \\
\text { de mes propres réflexions }\end{array}$ & 0 & 2 & $\mathbf{- 2}$ \\
\hline 11 & $\begin{array}{l}\text { C'est durant la pandémie que j'ai commencé à utiliser les réseaux sociaux } \\
\text { pour découvrir des outils numériques }\end{array}$ & -4 & -1 & $\mathbf{- 2 *}$ \\
\hline 13 & $\begin{array}{l}\text { Durant la pandémie, j'ai utilisé les réseaux sociaux pour des questions sur } \\
\text { la gestion de classe }\end{array}$ & -3 & -1 & $\mathbf{- 3}$ \\
\hline 1 & Avant la pandémie, j'utilisais un réseau social de façon sporadique. & -2 & -1 & $\mathbf{- 3}$ \\
\hline 8 & $\begin{array}{l}\text { Durant la pandémie, je n'ai pas fait appel aux réseaux sociaux plus } \\
\text { souvent qu'avant }\end{array}$ & 2 & -1 & $\mathbf{- 4 *}$ \\
\hline 15 & $\begin{array}{l}\text { Après la pandémie, j'ai l'intention d'utiliser moins souvent les réseaux } \\
\text { sociaux }\end{array}$ & 1 & -2 & $\mathbf{- 4 *}$ \\
\hline
\end{tabular}

Utilisateurs actifs des réseaux sociaux qui les inspiraient, en majeure partie pour le choix de solutions d'ordre pédagogique avant la pandémie, ils ont augmenté la fréquence d'utilisation durant la pandémie et n'envisagent pas la baisser après la pandémie (énoncés $5 ; 14 ; 17 ; 8 ; 15$ ). En plus des raisons de nature pédagogique, à la différence de leurs collègues du deuxième profil, ils ont fait appel aux réseaux sociaux durant la pandémie pour régler des problèmes techniques rencontrés lors de l'utilisation des outils numériques (énoncé 10).

Quant aux interactions avec les conseillers pédagogiques (rencontres virtuelles ou en présentiel, accompagnements individualisés ou centrés sur des problèmes de nature pédagogique), elles ne font pas partie de leurs options préférées (énoncés 29;30;28;30).

En guise de conclusion, trois profils professionnels d'enseignants se dégagent de notre recherche : les enseignants qui cherchent des solutions aux problèmes techniques sur Internet, ouverts aux relations de travail face à face avec les collègues et réticents aux échanges sur les réseaux sociaux (voir Facteur 1); les enseignants dont la pratique s'appuyait notamment sur les réflexions individuelles et les interactions avec les conseillers pédagogiques (voir Facteur 2); 
Revue internationale du CRIRES : innover dans la tradition de Vygotsky (2021) ISSN 2291-6717, vol 5, no 2, 95-111

Le développement professionnel autonome chez les enseignants dans le conteste de la pandémie

les utilisateurs actifs des réseaux sociaux (voir Facteur 3), soit les enseignants qui renonçaient à presque toute autre forme de collaboration que celle offerte sur les réseaux sociaux.

\section{Discussion et conclusion}

Pour répondre à notre question de recherche, nous avons exploré les ressources mobilisées par des enseignants œuvrant dans des écoles québécoises primaires ou secondaires avant, durant et après la crise due à la pandémie de la COVID. Deux ressources ont été explorées en profondeur, soit les réseaux sociaux et les conseillers pédagogiques. Complémentairement, nous avons conçu des énoncés qui reflètent l'apport des collègues, de l'Internet et des réflexions individuelles sur leur développement professionnel.

L'utilisation des réseaux sociaux par les enseignants précède le début de la pandémie; ils étaient utilisés notamment pour la découverte de nouveaux outils et pour des réponses aux questions d'ordre pédagogique. Plusieurs études, dont nous indiquons ici, à titre d'exemple, celles de Casey et Evans (2011) et Damani et Rinaudo (2011) témoignent dans ce sens. Ces chercheurs s'intéressaient bon nombre d'années avant la pandémie à l'apport des réseaux sociaux aux pratiques enseignantes et au processus d'apprentissage chez les élèves.

Comme le montrent nos résultats, qui concordent avec ceux des recherches menées par Ellis et al., (2020) et Luckerhoff et al., (2021), les épisodes de confinement, avec l'importance gagnée par le travail en ligne ou hybride, ont forcé les enseignants à repenser leur enseignement et la façon d'utiliser les médias socionumériques. En expliquant la raison de ces changements, Szirmai et al., (2020) affirment qu'un parcours d'apprentissage à distance et un suivi des tâches, en plus des supports de transmission de plus en plus sophistiqués, demandent aux enseignant l'adoption de stratégies mêlant le cognitif et l'affectif, et la production de consignes claires et de rétroactions efficaces.

Bouleversés par une crise pandémique non anticipée, les enseignants se sont sentis désemparés (Lollia \& Issaieva, 2020) et obligés d'adapter leurs pratiques afin d'assurer la continuité des apprentissages. Pour ce faire, la plupart ont fait appel aux ressources externes tandis que d'autres ont misé principalement sur leurs réflexions. Le choix des ressources semble être influencé par les expériences professionnelles antérieures à la période de crise et par l'activité envisagée : stratégie d'enseignement, gestion de classe, problème technique, etc.

Ainsi, le manque d'expérience sur les réseaux sociaux avant la période de confinement semble orienter les enseignants plus vers les conseillers pédagogiques alors que les contacts sur les réseaux sociaux commencés avant la pandémie se sont multipliés durant la période de confinement. Ces résultats ne sont pas surprenants étant donné l'ampleur et la vitesse de déclenchement de la crise sanitaire. Ces réalités ont obligé les enseignants à chercher des réponses rapides reliées davantage aux réflexions sur des connaissances tacites issues des expériences personnelles et fondées sur le dialogue avec le réel et sur la réflexion dans l'action et sur l'action (Schôn, 1987).

À partir de la définition d'Impedovo (2021, p.7) qui conçoit le développement professionnel « comme un processus intégré dans le temps présent, influencé par le passé et orienté vers le futur [...] », nous avons exploré l'intention des enseignants de faire appel aux réseaux sociaux après la crise sanitaire et leurs réponses ne font pas l'unanimité. Ainsi, si « les utilisateurs actifs des réseaux sociaux » (voir Facteur 3) pensent garder la même fréquence d'utilisation, les utilisateurs modérés (voir Facteur 1) expriment l'intention de diminuer la fréquence avec laquelle ils y font appel, tandis que les enseignants qui évitent les réseaux sociaux expriment leur intention 
de ne pas les utiliser moins souvent (voir Facteur 2). Le fait que la plupart des enseignants ont augmenté la fréquence d'utilisation des réseaux sociaux durant la pandémie et n'envisagent pas la diminuer une fois la période de confinement dépassée suggère des changements durables qui seront intégrés dans les pratiques quotidiennes. Dans ce contexte, le développement professionnel réside dans la transformation du fonctionnement psychique de l'apprenant, résultant de l'intégration de savoirs investis de sens (Buysse \& Vanhulle, 2009).

Le soutien de la part des conseillers pédagogiques représente la deuxième ressource du développement professionnel explorée. Les valeurs des tris de synthèse par facteur des dix énoncés font ressortir deux constats. Le premier est que, de façon générale, les enseignants qui évitent les réseaux sociaux misent plus sur l'expertise des conseillers pédagogiques lors des défis pratiques. Le deuxième réfère aux perceptions des enseignants face aux compétences attendues chez les conseillers pédagogiques et aux formes d'interaction. Ainsi, si les enseignants s'attendent que les conseillers pédagogiques maîtrisent des compétences technologiques, qu'ils partagent des capsules pratico-pratiques et offrent des formations en petits groupes et des réponses rapides à leurs questions, la plupart se montrent intéressés par les rencontres présentielles ou virtuelles. La diversité d'attentes mise en évidence par notre recherche pourrait venir d'un sentiment d'insécurité, un sentiment spécifique à une période de la crise (Duchesne, 2016). Selon la chercheuse, ce sont particulièrement les enseignants qui vivent de l'insécurité qui souhaitent le plus recevoir des outils et des ressources pédagogiques de la part des conseillers pédagogiques.

Rapportés à l'enseignement dans le contexte de la pandémie de la COVID-19 et concernés par la façon dont les enseignants ont fait appel aux différentes ressources de développement, nos résultats devraient servir aux conseillers pédagogiques pour mieux soutenir le développement de compétences permettant l'adaptation des pratiques enseignantes à des situations hors du commun. Par exemple, la présence des conseillers pédagogiques sur les réseaux sociaux pourrait apporter des pistes d'accompagnement et même guider les échanges des enseignants qui y sont.

En ce qui a trait à la question de recherche, nous tirons la conclusion que les médiations entre des acteurs de l'éducation durant la pandémie de la COVID-19 ont permis le développement professionnel chez les enseignants travaillant dans des écoles primaires et secondaires québécoises. Opérationnalisé ici à partir des changements de pratiques dus à l'acquisition ou au renforcement des compétences dans le contexte de la pandémie due au COVID 19, le développement professionnel s'avère un processus complexe qui repose sur la confiance dans les habiletés de réfléchir, ainsi que sur l'engagement dans des interactions professionnelles, virtuelles et en présence.

Les expériences professionnelles et la nature du problème à résoudre ont eu davantage d'influence que l'ordre d'enseignement, l'ancienneté (générale ou dans la tâche actuelle) sur le choix des formes privilégiées de développement professionnel durant la pandémie : réseaux sociaux, conseillers pédagogiques, collègues, Internet ou autoréflexions.

Notre recherche comporte évidemment certaines faiblesses et limites. Tout d'abord, il y a la subjectivité des participants qui s'étend à la comparaison des énoncés, les uns par rapport aux autres à l'occasion de leur classement. Puis, « le chercheur apporte sa part de subjectivité par la constitution du répertoire des énoncés : le choix, la formulation et l'organisation autour de thèmes prédéfinis [...]» (Dobrica-Tudor \& Théorêt, 2017, p. 129). La principale limite est l'absence d'entretiens avec les participants qui auraient pu aider à une meilleure compréhension de la logique qui se trouve derrière les classements et des potentielles limites comme l'accès à la technologie, les connaissances et l'aisance concernant son utilisation. 
Revue internationale du CRIRES : innover dans la tradition de Vygotsky (2021) ISSN 2291-6717, vol 5, no 2, 95-111

Le développement professionnel autonome chez les enseignants dans le conteste de la pandémie

\section{Références}

Berten, A. (1999). Dispositif, médiation, créativité : Petite généalogie. Hermes, 25, 33-47. https://doi.org/10.4267/2042/14972

Buysse, A. \& Vanhulle, S. (2009). Écriture réflexive et développement professionnel : quels indicateurs? Questions Vives, 5 (11), 225-242. https://doi.org/10.4000/questionsvives.603

Carpenter, J. P., \& Krutka, D. G. (2015). Engagement through microblogging: Educator professional development via Twitter. Professional Development in Education, 41(4), 707-728. https://doi.org/10.1080/19415257.2014.939294

Casey, G. \& Evans, T. (2011). Designing for Learning: Online Social Networks as a Classroom Environment. International Review of Research in Open and Distributed Learning, 12(7), 1-26. https://doi.org/10.19173/irrodl.v12i7.1011

Dariel, O. (2013). La méthode Q, une méthodologie innovante pour la recherche infirmière. Soins ; la revue de référence infirmière, 58 (776), 55-60. doi : 10,101 6/j.soin.2013.04.015

Damani, K. \& Rinaudo, J-L. (2011). Enseigner avec les réseaux sociaux : des professeurs sur facebook. EPAL - Echanger pour apprendre en ligne, Grenoble, France. ffhal-02010592f

Desgagné, S. \& Bednarz, N. (2005). Médiation entre recherche et pratique en éducation : faire de la recherche «avec » plutôt que «sur» les praticiens. Revue des sciences de l'éducation, 31 (2), 245-258. https://doi.org/10.7202/012754ar

Dobrica-Tudor, V. \& Théorêt, M. (2017). La méthode Q dans l'étude des réflexions des enseignants du secondaire sur leurs pratiques professionnelles. Mesure et évaluation en éducation, 40 (2), 125-153. https://doi.org/10.7202/1043570ar

Duchesne, C. (2016). Complexité et défis associés aux rôles de conseiller pédagogique. McGill Journal of Education/Revue des sciences de l'éducation de McGill, 51 (1), 635-656. https://doi.org/10.7202/1037363a

Durat, L. \& Kern, D. (2019). Accompagner la réflexivité sur l'expérience : une médiation cognitive et socioaffective vers l'apprentissage. Activités [En ligne], 16 (1). Consulté le 18 septembre 2021 sur URL http://journals.openedition.org/activites/4027

Duval, S., Lehrer, J., Pirard, F., \& Housen, M. (2021). La pandémie, source de risques et d'opportunités pour la mise en place de pratiques de transition collaboratives lors de l'entrée à l'école. Revue Internationale Du CRIRES : Innover Dans La Tradition De Vygotsky, 5 (1). https://doi.org/10.51657/ric.v5i1.41066

Ellis, V., Steadman, S., \& Mao, Q. (2020). «Come to a screeching halt»: Can change in teacher education during the COVID-19 pandemic be seen as innovation? European Journal of Teacher Education, 43(4), 559-572. https://doi.org/10.1080/02619768.2020.1821186

Ellingsen, I. T., Stephens, P. \& Størksen, I. (2012). Congruence and incongruence in the perception of "family" among foster parents, birth parents and their adolescent (foster) children. Child and Family Social Work, 17, 427-437. https://doi.org/10.1111/J.1365-2206.2011.00796.X

Ferry, J.-M. (2014). Approches différentielles de la notion de crise. Revue économique et sociale : bulletin de la Société d'Études Économiques et Sociales, 72 (3-4), 44-54.

Gauzente, C. (2013). Une invitation illustrée à utiliser la Q-method dans les Recherches en Systèmes d'Information. Systèmes d'information \& management, 18(2), 69-109. https://doi.org/10.3917/sim.132.0069

Ghouname, N. (2020). Moodle or Social Networks: What Alternative Refuge is Appropriate to Algerian EFL Students to Learn during Covid-19 Pandemic. Arab World English Journal, 11 (3): 21-41. https://dx.doi.org/10.24093/awej/vol11no3.2 
Revue internationale du CRIRES : innover dans la tradition de Vygotsky (2021) ISSN 2291-6717, vol 5, no 2, 95-111

Le développement professionnel autonome chez les enseignants dans le conteste de la pandémie

Granger, N., Guillemette, S. et B-Lamoureux, B. (2021). La fonction de conseillère ou de conseiller pédagogique au Québec. Rapport de recherche. Université de Sherbrooke, Canada.

Impedovo, M. A. (2021). Développement professionnel agentif et collaboratif des enseignants : communautés épistémiques via les réseaux sociaux en temps de crise. Revue internationale du CRIRES: innover dans la tradition de Vygotsky, 5 (1). https://doi.org/10.51657/ric.v5i1.41069

Kuhne, N., Abernot, Y. \& Camus, D. (2008). Le Q-sort, un outil pour la recherche en soins : le cas des représentations chez les infirmiers en psychiatrie de l'âge avancé. Recherche en soins infirmiers, 95 (4), 46-56. https://doi.org/10.3917/rsi.095.0046

Lameul, G., Peltier, C. \& Charlier, B. (2014). Dispositifs hybrides de formation et développement professionnel. Effets perçus par des enseignants du supérieur. Éducation \& Formation, e301, 99-113. https://archive-ouverte.unige.ch/unige: 37228

Lenoir, Y. (1996). «Médiation cognitive et médiation didactique». Dans C. RAISKY \& M. CAILLOT (dir.), Le didactique au-delà des didactiques : regards croisés sur des concepts fédérateurs (pp.223-251). Bruxelles : De Boeck Universitaire.

Liljekvist, Y.E., Randahl, A-C., van Bommel, J. et Olin-Scheller, C. (2021). Facebook for Professional Development: Pedagogical Content Knowledge in the Centre of Teachers' Online Communities. Scandinavian Journal of Education Research, 65 (5), 723-735. https://doi.org/10.1080/00313831.2020.1754900

Lefeuvre, G., Garcia, A., \& Namolovan, L. (2011). Les indicateurs de développement $\begin{array}{lllll}\text { professionnel. } & \text { Questions } & \text { 277-314. }\end{array}$ http://dx.doi.org/10.4000/questionsvives.627

Liou, Y-H \& Canrinus, E. (2020). A capital framework for professional learning and practice. International Journal of Educational Research, 100. https://doi.org/10.1016/j.ijer.2019.101527

Lollia, M., \& Issaieva, E. (2020). Comment les enseignants assurent la continuité pédagogique et évaluent en contexte de pandémie? Une étude en Guadeloupe. Évaluer. Journal international de recherche en éducation et formation, Numéro Hors-série, 1, 181-192.

Luckerhoff, J., Raynauld, V. \& Lalancette, M. (2021). Note de recherche : du désintéressement à l'emballement: les médias socionumériques pour enseigner, diffuser, communiquer et recruter dans le milieu universitaire. Enjeux et société, 8 (2), 323-343. https://doi.org/10.7202/1078500ar

Macià, M., \& García, I. (2016). Informal online communities and networks as a source of teacher professional development: A review. Teaching and Teacher Education, 55, 291-307. http://dx.doi.org/10.1016/j.tate.2016.01.021

Mashhadi, F., Biria, R., \& Lotfi, A. (2020). From sociocultural epistemology to transformative pedagogy: Contributions of collaborative action research through dialogic mediation in teacher professional development. Journal of Modern Research in English Language Studies, 7(2), 51-75. http://dx.doi.org/10.30479/jmrels.2020.12104.1506

Ramlo, S. \& John, N. (2020). Divergent student views of cybersecurity. Journal of Cybersecurity Education, Research and Practice, 2019 (2), Article 6. Available at: https://digitalcommons.kennesaw.edu/jcerp/vol2019/iss2/6

Rioux-Dolan, M. (2004). L'accompagnement du personnel enseignant : enjeux et défis dans le contexte de la réforme. Dans M. L'Hostie et L.P. Boucher (dir.). L'accompagnement en 
Revue internationale du CRIRES : innover dans la tradition de Vygotsky (2021) ISSN 2291-6717, vol 5, no 2, 95-111

Le développement professionnel autonome chez les enseignants dans le conteste de la pandémie

éducation: un soutien au renouvellement des pratiques (pp.13-30). Québec: Presses de l'Université du Québec. ISBN 2-7605-1278-9

Schön, D.A. (1987). Educating the reflective practitioner: Toward a new design for teaching and learning in the professions. San Francisco: Jossey-Bass. ISBN 1-55542-025-7

Shriki, A. (2021). Facebook groups as a space for responding professional needs of teachers during the COVID-19 period. EDULEARN21 Proceedings 13th International Conference on Education and New Learning Technologies July 5th-6th, 2021. ISBN: 978-84-09-31267-2

Stone, C. \& Springer, M. (2019). Interactivity, connectedness and "teacher-presence": Engaging and retaining students online. Australian Journal of Adult Learning, 59(2), 146-169

Szirmai, M., Birsan, C., Lemay, D., Ouvrard, L., Stockinger, P., Vigny, P.-J., Tice-Dsirn, U., Herve, R. (2020). Enseigner à distance au pays du soleil levant et des hautes technologies. Hal-03117 451 v1

Tardif, M. \& Lessard, C. (2004). Le travail enseignant au quotidien (2e éd.). St-Nicolas, QC : Presses de l'Université Laval. ISBN 2-7637-7653-1

Vérin, A., \& Peterfalvi, B. (1985). Un instrument d'analyse des modèles implicites de l'enseignement scientifiques chez enseignants. Aster. Recherches en didactique des sciences expérimentale, 1, 7-28. 
Revue internationale du CRIRES : innover dans la tradition de Vygotsky (2021) ISSN 2291-6717, vol 5, no 2, 95-111 Le développement professionnel autonome chez les enseignants dans le conteste de la pandémie

\section{Annexe}

\section{Matrice des contributions des Q-sorts aux facteurs}

\begin{tabular}{|c|c|c|c|}
\hline Q SORT & Facteur 1 & Facteur 2 & Facteur 3 \\
\hline 1 & $0.5981 X$ & 0,4735 & $-0,0243$ \\
\hline 2 & 0,2769 & 0,1287 & 0,3189 \\
\hline 3 & $0.4684 X$ & $-0,0784$ & 0,3451 \\
\hline 4 & $0.4660 X$ & 0,2516 & 0,1403 \\
\hline 5 & $-0,1522$ & $0.5278 X$ & 0,2002 \\
\hline 6 & $0.7034 X$ & $-0,0163$ & 0,0843 \\
\hline 7 & $-0,0426$ & 0,1649 & $0.5169 X$ \\
\hline 8 & $-0,2938$ & 0,1596 & $0.5555 X$ \\
\hline 9 & $0.7049 \times$ & $-0,0420$ & 0,1077 \\
\hline 10 & 0,2257 & $-0,2393$ & $0.5745 X$ \\
\hline 11 & $-0,1545$ & 0,3923 & $0.5755 X$ \\
\hline 12 & 0,1372 & $0.6982 X$ & $-0,1507$ \\
\hline 13 & 0,1452 & 0.7183 X & 0,0410 \\
\hline 14 & 0,1452 & 0,0278 & $0.3619 X$ \\
\hline 15 & $-0,2097$ & $0.5141 X$ & $-0,2061$ \\
\hline 16 & $0.6600 \times$ & 0,3635 & 0,2916 \\
\hline 17 & 0,0229 & 0,0296 & $0.4660 X$ \\
\hline 18 & $0.7064 X$ & $-0,0059$ & 0,2043 \\
\hline 19 & 0,4660 & 0,1020 & 0,0591 \\
\hline 20 & 0,1932 & $-0,0393$ & $0.7418 X$ \\
\hline 21 & $0.4673 X$ & 0,0400 & 0,0088 \\
\hline 22 & 0,2184 & $0.6261 X$ & 0,3423 \\
\hline 23 & 0,3699 & 0,0618 & $0.5839 X$ \\
\hline 24 & 0,1363 & $0.4906 \times$ & 0,2626 \\
\hline 25 & 0,0245 & $0.6181 X$ & 0,1807 \\
\hline 26 & 0,4346 & $-0,0434$ & $0.5642 \times$ \\
\hline 27 & 0,4164 & 0,2603 & $0.5127 X$ \\
\hline 28 & 0,0043 & 0,1666 & 0,2834 \\
\hline 29 & 0,1004 & $0.6903 X$ & $-0,0028$ \\
\hline Valeur propre & 6,1 & 3,2 & 2,5 \\
\hline \% Expl. Var. & 24 & 17 & 17 \\
\hline Fiabilité & 0,97 & 0,97 & 0,976 \\
\hline
\end{tabular}

